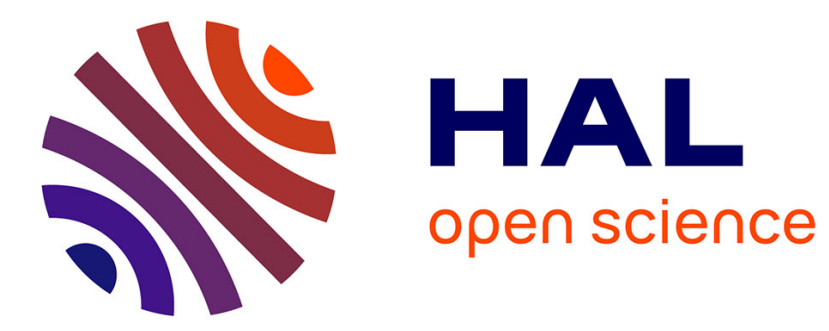

\title{
" Errare Humanum Est": simulation of communication error among a virtual team in crisis situation
}

\author{
Lauriane Huguet, Nicolas Sabouret, Domitile Lourdeaux
}

\section{To cite this version:}

Lauriane Huguet, Nicolas Sabouret, Domitile Lourdeaux. " Errare Humanum Est ": simulation of communication error among a virtual team in crisis situation. 15th IEEE International Conference on Cognitive Informatics and Cognitive Computing (ICCI*CC 2016), Aug 2016, Palo Alto, CA, United States. pp.344-350. hal-01356193

\section{HAL Id: hal-01356193 \\ https://hal.science/hal-01356193}

Submitted on 25 Aug 2016

HAL is a multi-disciplinary open access archive for the deposit and dissemination of scientific research documents, whether they are published or not. The documents may come from teaching and research institutions in France or abroad, or from public or private research centers.
L'archive ouverte pluridisciplinaire HAL, est destinée au dépôt et à la diffusion de documents scientifiques de niveau recherche, publiés ou non, émanant des établissements d'enseignement et de recherche français ou étrangers, des laboratoires publics ou privés. 


\title{
“Errare Humanum Est": simulation of communication error among a virtual team in crisis situation
}

\author{
Lauriane Huguet* ${ }^{\dagger}$, Nicolas Sabouret ${ }^{\dagger}$ and Domitile Lourdeaux* \\ * Sorbonne universités, Université de technologie de Compiègne, CNRS, Heudiasyc UMR 7253, \\ 60203 Compiègne Cedex \\ ${ }^{\dagger}$ Limsi, CNRS, Univ. Paris Sud, Université Paris Saclay, F - 91405, Orsay \\ Email: lauriane.huguet@hds.utc.fr,nicolas.sabouret@limsi.fr,domitile.lourdeaux@hds.utc.fr
}

\begin{abstract}
In the context of medical team leaders training, we present a multiagent communication model that can introduce errors in a team of agents. This model is built from existing work from the literature in multiagents systems and information science, but also from a corpus of dialogues collected during actual field training for medical teams. Our model supports four types of communication errors (misunderstanding, misinterpretation, non-understanding and absence of answer) that appear at different stages of the communication process.
\end{abstract}

\section{Introduction}

First-aid worker's job is evolving: the interventions in urban zones with a civilian population, as well as in the military context, often take place on unsecured grounds or with too many victims compared to the available resources. These crisis situations are sources of stress [1] and thus are more error prone, as showed by [2]. To manage this stress, it is necessary to be prepared to face various "exceptional" situations in a way that a number of unforeseen situations [3] become possible situations (of the same kind as those they have already been faced to, in theory or in practice) and are no longer unminded situations [4].

That is why, in the rescue teams training context, the French firefighters of Paris or the military medecine school (Ecole du Val de Grâce) organize "life-size" simulations. These trainings require the intervention of numerous operators and mobilizes a lot of equipments. Therefore, they cannot be used at a large scale. The VICTEAMS project ${ }^{1}$ aims at creating a virtual environment for training rescue team leaders to such crisis situation. In this environment, the user is a team leader that interacts with a team of virtual autonomous first-aid workers. The use of a virtual environment allows not only tutors to increase the frequency of the trainings, but also learners to face situations which cannot be set in real-world simulation.

The VICTEAMS project focuses on the training of non-technical skills in Health care delivery. Non-technical skills are the cognitive skills, social skills and professional resources which complete the technical skills of a person

1. VIrtual Characters for team Training: Emotional, Adaptive, Motivated and Social. https://victeams.hds.utc.fr/

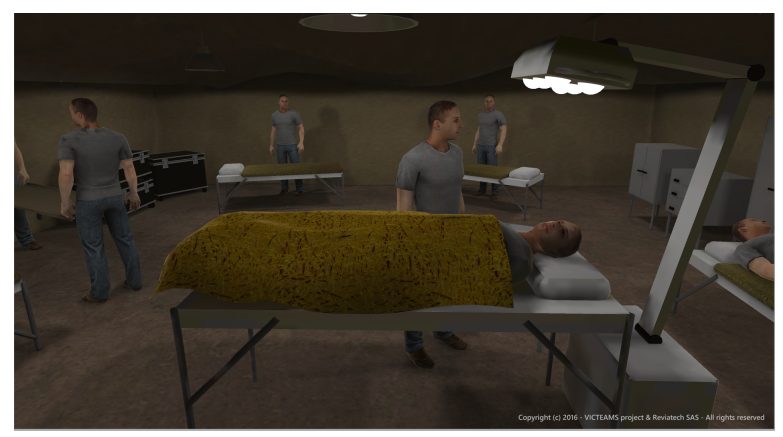

Figure 1. The virtual environment of the VICTEAMS project.

and contribute to the safety and to the better efficiency in the execution of the task. Indeed, studies [5] showed that more than $70 \%$ of the medical errors are due to errors connected to the non-technical skills and more particularly to the communication within the medical team.

In this context of training, our objective in this paper is to propose a model of communication able to reproduce within a simulator the communication errors as they occur in a real medical team. To this goal, we study the various types of errors that can occur and at which stage they appear in the communication process. For example, we build agents that can forget to communicate a crucial piece of information, or do not transmit it on the right time, or interpret the messages they receive in a different way as intended by the sender, etc.

The next section presents a brief review of literature. We shall then present our model that integrates communication errors (section 3 ) followed by a preliminary evaluation based on a questionnaire (section 4).

\section{Related Work}

For our work, we were brought to study several research domains around communication: the theories of communication in signal processing $\&$ in language sciences on the one hand, and the computational models of communication on the other. 


\subsection{Communication Theory}

One of the first formal model of communication comes from Shannon [6] who proposes a mathematical model of the communication. He states that a message is encoded then sent through a certain channel to a recipient who decodes it. To guarantee the integrity of the message it is thus important that the channel is not noisy and that the coding of the information is shared by the sender and the receiver. These elements are interesting to understand where we can introduce communication errors: at the encoding level, at the level of the environment which conveys the message and at the level of the receiver who decodes the message.

At the reception of the message, we find three types of error defined by Mac Roy [7].

1) non-understanding: The received message does not make sense and the sense cannot be repaired.

2) misinterpretation: The message is meaningful but disagrees with our knowledge.

3) misunderstanding: We think we understood the received message but what is understood is different than what was sent.

By studying a set of videos collected with military medical officers, we were able to observe these various types of error in-situ. In the first case, the error on the message is syntactic, the message is too much degraded to be accepted (for example, a helicopter flies over the scene when the operator announces the delay before the evacuation). The receiver has no choice but to ask for the information to be repeated.

In the second case, the message raises a semantic problem to the receiver because it goes against his or her own knowledge. For example, when the medical leader asks the nurse about the arm of the victim and the nurse does not understand the question. The nurse had no information of a pain in the arm of the patient and had focused on the other traumas brought to her attention.

Finally in the third case, the receiver thinks he or she has understood correctly the message but his/her understanding is different from what the sender intended. The error is not always immediately visible and it is even sometimes possible not to realize it. In one of our video, the team leader asks a nurse if one has to start a coniotomy procedure in an affirmative way (in French) and the nurse understood it as an order and not as a question, so she simply answers "yes" (and assumes the operation has to be conducted). This error was detected only during the debriefing.

This third type of errors is interesting because it shows that a message can be understood in different ways by the interlocutors. The square model of [8] gives an interesting frame to conceptualize this phenomenon. The principle is that a message always carries four components: a fact (content), a self-revelation, a relationship clue and an appeal to action. These four components are always present in the message, but in a more or less stressed way and more or less deliberate way. Thus, the receiver can interpret a message in a different manner than what was intended by the sender, because both interlocutors do not give the same importance to the various components of the message. According to [9], this is what leads to misunderstanding.

This idea that a message can be understood in several ways is essential to our model. Yet, it seems that it is very hard to get exactly four meanings corresponding to the four proposed components of the square model. This is the reason why we adapted this model to our needs.

\subsection{Computational model of communication}

The study of communication theory allows us to stress some possibility to introduce errors in communications. To obtain a computational model, we also have to look at the computer science approach to this question, especially in multiagent systems.

2.2.1. Speech or Dialogue generation. In computer science, the communication models are based on the speech act theory that has been developed by Austin [10] and Searle [11]. This theory distinguishes the locutionary act (the expression of the message in natural language), the illocutionary act (the meaning of the sent message) and the perlocutionary act (the effect of the message on the receiver). To describe this illocutionary and perlocutionary acts, a message is formed of a performative that represent the goal of communication, for example "Inform" or "Order" and a content that defines the topic of the message.

In the area of multiagent systems, the FIPA standard [12] is widely used for message representation. This standard proposes that a communication can only be successful if the illocutionary and the perlocutionary are the same. Therefore there is no communication error possible at this level: the messages sent or received are identical. Furthermore, the standard FIPA defines communication protocols which force the order in which messages can be received and the way to interpret them. The goal is to minimize the errors.

That is also the case in dialogue systems, whose goal is also to produce a relevant statement in the conversational context, whether it is by reactive planning [13] or by mixed initiative [14]. To obtain a good communication within the framework of the human-agents communication in virtual environment is already a difficulty because it is necessary to support all the types of interactions (human-agent or agentagent) and because all the messages should be interpretable without losing freedom or expressiveness [15].

Thus, the optimization of communication with the aim of an effective cooperation has been the main point of interest of the community. On the contrary, our work tries to introduce errors in a convenient and explicable way into the communication. Our aim is to raise awareness of the user to the consequences of the errors of communication.

2.2.2. Communication errors processing. A few research in automated language processing [16] focused on the study of communication errors and in particular on syntactical errors. The derived models study are essentially statistical [17], [18]: they focus on the error rate at the lexical or 
syntactic level and generally do not account for the context of the interaction.

Yet the consideration of the context is important in our model to include the errors at the semantic level. A frequently observed error is to exchange the terms such as "morphin" and "adrenalin". These terms are similar at the semantic level because they are both medical terms, they both refer to substance that can be injected and both are frequently used by the medical team members. However their uses are opposite and confusion between both has heavy consequences on the victims. The confusion which seems lexical is in fact a confusion at the semantic level bound to the medical context. That is why we propose a semantic model capable of reproducing this type of communication errors.

In dialogue systems, there also exists work that propose communication management algorithms to repair "misinterpretation" errors [19]. However, they do not propose, to our knowledge, models to produce these errors to be repaired.

In our proposal bellow, we describe the first version of our communication model that supports the injection of errors at each stage of the communication (generation, transmission and reception).

\section{Proposal}

\subsection{Overview of the model}

In our virtual environment populated with interacting autonomous agents. Every agent is characterized by a name, a role (leader, transmission operator, ...) and a status (doctor, (male) nurse, ...) allowing it to be call out. Among the state variables of the agent, we shall be interested more particularly in its position in the virtual environment and its looking direction: both will be used in section 3.3). We also use a variable of "stress" which takes its value in $[0,1]$ and which allows us to control the rate of error (see section 3.5.3). We also add a personal threshold that represents the maximum level of stress the agent can handle:

Agent $=\langle$ Name, role, status, position, orientation, threshold_stress, stress $>$

As it is classically done in multiagent system and as we illustrate on figure 2 , every agent that wants to communicate with other agents builds a message (cf. (1.), on figure 2), sends it through the environment which passes it on to the agents (cf. (2.), on figure 2). The transport of messages is done within an active environment [20] allowing the alteration of messages and the delivery to other agents than the initial recipient. Finally, every agent chooses to ignore or to treat the messages it receives (cf. (3.), on figure 2).

\subsection{Structure of a message}

3.2.1. Illocutionary act. The structure of a message is based on the speech act theory [11]: it includes the sender, the recipients and the information of the illocutionary act



Figure 2. Model scheme

as a couple (performative, content). The recipients are defined by their name, their role or their status. It is also possible not to specify recipients in a message (it is then broadcasted). The performatives, which characterize the treatment associated with the message, are application dependent: they can be chosen for example among those defined by the standards FIPA [12] or DIT++ [21], but it is possible to define other performatives appropriate to the considered application. In our application, we use four performatives described on figure 3 . The message contents are SPARQL queries [22] or RDF-triples [23].

As an example, to inform about the state of a patient we use the performative INFORM and of a set of RDF-triples:

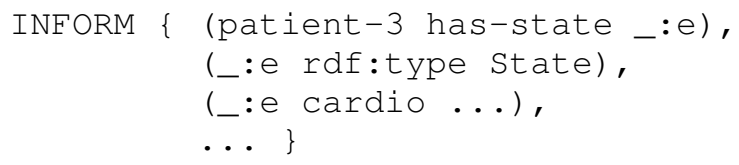

To ask for the time of the evacuation, we use the illocutionary act:

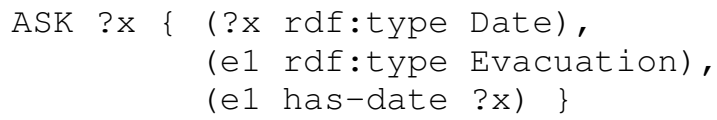

\begin{tabular}{|l|l|}
\hline Performative & Use \\
\hline Inform & Transmit a piece of information \\
\hline Ask & Ask about piece of information \\
\hline Order & Order an action \\
\hline Call & Draw the attention of an agent \\
\hline
\end{tabular}

Figure 3. Performatives used in the VICTEAMS project.

3.2.2. Perlocutionary act. A message can carry multiple interpretations. For example, in French, it is possible to ask a question in a affirmative way: there exists an ambiguity at the level of the performative. Also, there exists ambiguities of content. For example, in a sentence like "put it here", "it" might indicate a different object for the sender than for the receiver. To reproduce this phenomenon, the illocutionary act is completed by other couples (performative, content), symbolizing possible perlocutionary acts, as was proposed by the square model [8] presented earlier. 
We do not present here automatic mechanism to generate these perlocutionary acts, because it is a difficult problem which we have to take on. In the current version of the system, these acts are manually defined and in advance for the various types of misinterpretation occurring in real situations. These situations were extracted from a corpus of communications collected during trainings at the French military medical school École du Val de Grâce. We were able to identify two categories of errors. The first, often bound to the context, concerns the semantic contents, as in the example of exchange between adrenalin and morphine. This one occurs if we speak about adrenalin while there is a morphine syrette ready to be used, for example. It is thus the physical closeness of the object or the fact that we just used it that creates the confusion.

The other category of observed errors, as said before, concerns the performative (take a question for an assertion or the opposite). We observed that this type of error is strongly connected to the locutionary act. Thus we chose to suggest systematically the same contents with the two others performatives of the list (INFORM,ASK, ORDER), the case of the performative CALL being a little different (it is just used to draw the agent's attention).

3.2.3. Modality. On top of the previous elements, each part of the message is associated with a modality. For example, let us consider a first-aid worker who tells his team-mate "Give me ... [Gesture indicating the first-aid kit]". The message is complete but it consists of a verbal first part and a visual second part. The contents are thus split in couples $(c, m)$ where $c$ is a set of RDF-triples, subset of the global contents of the message, and $m$ the associated modality.

So, in our communication model, a message $M s g$ is composed of a set of meanings $M$, a sender $S$, and a set of recipients $A$ :

$$
M s g=(\{\text { Meanings }\}, S,\{A\})
$$

A meaning $M$ is a possible perlocutionary act. It is constituted by a performative $P$ and a set of couples (content, modality).

$$
M=(P,\{\text { (content, modality })\})
$$

The message created is then sent to the environment.

\subsection{Transport and alteration of a message}

The environment has a dual function in our model: it determines which agents are going to receive each message and with which degree of degradation. The degradation of the message can vary according to the way it is transmitted and can be different between the various receivers of the message.

If the different part of a message content are transmitted by different channels, it is possible to delete from the message only the contents passing through some channels. For example, if the first-aid worker tells his colleague "Give me [movement indicating the first-aid kit]" while the colleague is looking at the patient, the colleague will not see the visual part of the message and will thus receive "Give me ..." which is an incomplete message.

The people able to perceive a message are the ones verifying the conditions of transmission for the used modalities. For each existing agent $a$, the environment determines if the agent is able to receive the message $M$ sent by the sender according to the state of the transmission channels for every used modalities $\left\{\right.$ state $\left._{\bmod }\right\}$. Then it builds an altered message $M^{\prime}$ from $M$ :

$$
M^{\prime}=\operatorname{perceived}\left(M, a,\left\{\text { state }_{\text {mod }}\right\}\right)
$$

For example if the modality is visual, the message will be delivered to the agents positioned at the right place and looking in the right direction; if the modality is vocal, the message will be delivered to the agents close enough and who are not affected by loud background noise.

The agents that receive the message are thus not necessarily in the list of the recipients of the message, because the environment sends a message, more or less altered, to all the agents who satisfy the conditions of transmission of at least one of the used modalities. This allows us to reproduce the phenomenon of overhearing [20], [24]. This phenomenon allows an agent 1) to answer a question which is not intended for it, in case it considers it knows the answer, or 2) to deduce new information from conversations between other agents, what would not be the case if the message was delivered only to the deliberate recipients.

\subsection{Reception of a message}

Every agent within reach of the message receives is more or less altered message $M^{\prime}$ and decides if it ignores it or if it considers it. If the agent decides to process the message, it has to choose a perlocutionary act, that is one of the meanings contained in the message $M^{\prime}$, and to act accordingly, as indicated on point (4.) on figure 2 .

The table on figure 4 summarizes the various stages of model with the types of errors generated at every step and the functions proposed for these steps. Next section will give details on each of these functions.

\begin{tabular}{|l|l|l|}
\hline step & type of error & comments \\
\hline$(1)$. & misunderstanding & $\begin{array}{l}\text { production of alter- } \\
\text { native meanings }\end{array}$ \\
\hline$(2)$. & non-understanding & Algorithm 1 \\
\hline$(3)$. & absence of answer & Algorithm 2 \\
\hline$(4)$. & misinterpretation & Algorithm 2 \\
\hline
\end{tabular}

Figure 4. Summary of the errors supported by the model

\subsection{Implementation}

To implement the mechanisms presented above, we define three main functions, as shown on figure 5: 
- For the distribution and the alteration of the message by the environment, we use a function perceived which takes in argument a message and an agent and determines if the agent receives the message or not, and with which alterations.

- The function level_alteration allows an agent to determine a posteriori the level of alteration of a received message.

- The function acceptance allows an agent, according to the level of degradation and its own internal state (in particular the level of stress), to decide if it must process the message, ask the sender to send the message again or simply ignore the message.

These three functions are detailed in the following paragraphs.

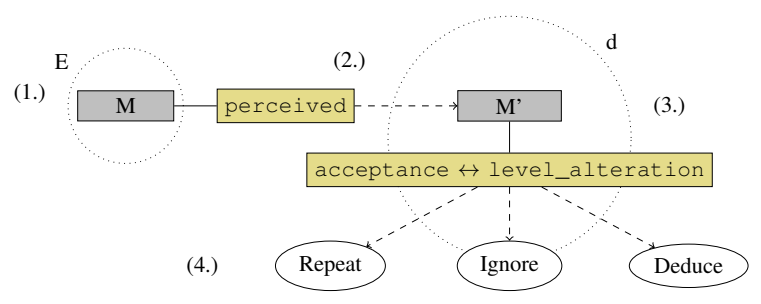

Figure 5. Steps in the treatment of a message

3.5.1. Perception of a message and alteration by the environment. All the messages, independently of the explicit recipients, are potentially transmitted to all agents, according to the principle of overhearing. So, for message $M$ and for each agent $a$, the environment builds an altered message $M^{\prime}(a)$ which defines the message received by the agent $a$. Two particular cases are to be considered. If the message could not be transmitted by any channel between the sender and $a$, then it is not distributed to the agent $a$. The other case is the one where the message was transmitted with an empty content. For example, let us imagine two medical team members who see each other but use a radio to communicate. If one of the medical team member sees the other one speaking on the radio and the radio is broken, he will receive a message but with empty content.

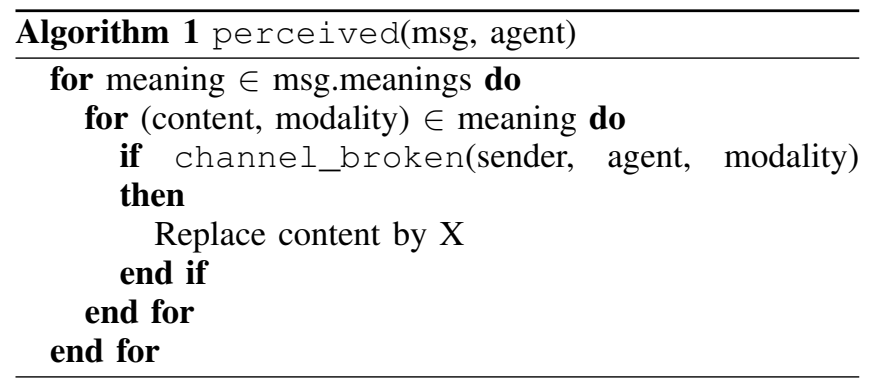

The function channel_broken takes in parameter two agents and a modality and returns True if the channel conveying the modality between both agent is broken.
When part of the message cannot be perceived by the agent who receives the message, we replace the corresponding content by a keyword X. It allows us to spot the damaged parts of the message for the computation of the alteration level presented below.

3.5.2. Computation of the alteration level. In the current version of the model, the computation of the degradation of the message is a simple computation of the rate of missing contents. There is thus no semantic information about the alteration of the message. As explained in the previous section, the computation is made possible by the replacement of the unperceived contents by the keyword $\mathrm{X}$.

$$
\text { level_alteration }(\mathrm{msg})=\frac{\text { number of } \mathrm{X}}{\text { number of triples }}
$$

This computation of alteration is not optimal because it is possible that some alteration occurs on unnecessary information and that the received part is enough to treat the message. However, we wanted our agents to adopt a careful behaviour when in an unstressed situation. Eventually, we intend to define a more complete mechanism to compute the alteration level which account for the context.

3.5.3. Reception of a message: When to make the sender repeat. Based on a review of literature in human sciences, it seems that the more stressed we are, the more the attention focuses [25] (we speak of tunnelling). Our function of acceptance of messages decomposes into two parts. If the level of stress of the agent is higher than a threshold private to the agent then there is a high probability $\alpha$ that it ignores the received message (the parameter $\alpha$ is to be calibrated). Otherwise, we lean on the assertion of [2] which explains that the more the agents are put under stress, the more they are prone to errors (that is the opposite of what it should make in optimal situation). So, when the agent is put under stress (but under the threshold of tunnelling), it should ask to repeat the good messages more than the bad ones. When the agent is not stressed, the probability to ask to repeat a message is proportional to its alteration level.

This point thus brings us to choose a "saddle function" shown on figure 6 . Thanks to an interpolation and by taking into account previously expressed constraints, we obtained the equation:

$$
\text { repeat }(s, d)=0.625 s^{2}-1.55 s d+0.125 s+0.95 d+0.04,
$$

where $s$ is the stress level of the agent and $d$ is the alteration.

The acceptance function, presented on figure 2 , indicates, depending on the received message and the agent's stress level, whether the agent ignores the message which it receives, asks the speaker to repeat or decides to act according to what it can deduce.

\section{Preliminary study}

The production of alternative meanings described in the previous section should support the generation of a large 


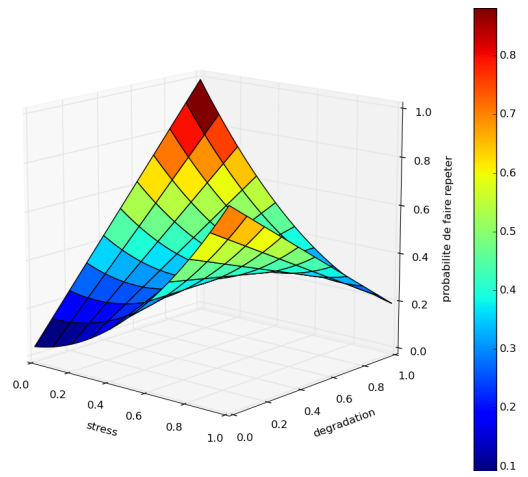

Figure 6. Graph of the function repeat(stress, alteration)

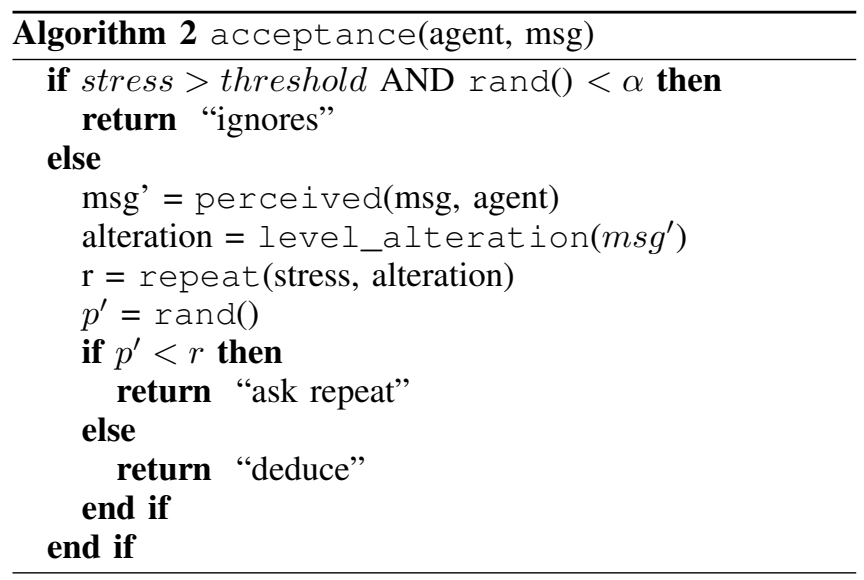

number of possible messages. We have to select a small number of them (one or two) to be sent to the recipient agent, so as to avoid that the communication mechanism overwhelms the global system. Moreover, all proposed solutions do not have the same technical difficulty.

This is the reason why we need to evaluate in advance which errors can appear in our virtual environment and with which probability. Since the interaction will be text-based, we cannot rely on our (verbal) data field corpus. We used a questionnaire to extract this information.

The questionnaire proposes locutionary messages without punctuation (to avoid bias on the message interpretation). Participants were asked to rate in a 0 to 4 scale several possible alternative meanings that were proposed for each message, with the additional constraint to answer as quickly as possible. It was allowed to propose another alternate meaning if necessary.

This questionnaire has been filled-in by 16 participants. While this is not enough to claim for any valid result from a statistical point of view, this gave us some insights on the model's requirements. First, there is very little consensus on the meaning, as illustrated on figure 7. This advocates for having several alternative meanings for one single message.

We could also confirm that the semantic error ("adrenaline" instead of "morphine") appears quite often (figure 8). This is why our end goal, is to generate these sort

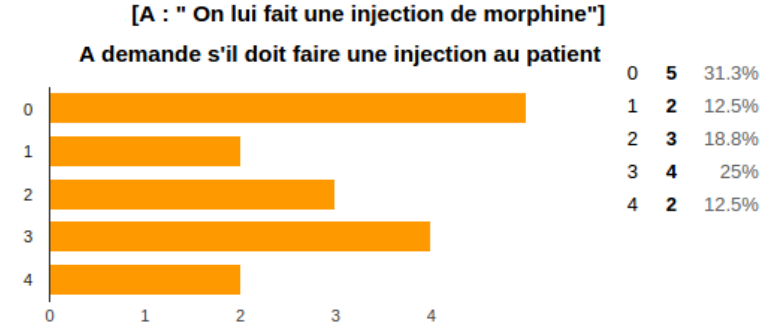

Figure 7. Example where participants disagree - misunderstanding

of alternative meanings using semantic distance measures in domain ontologies [26].

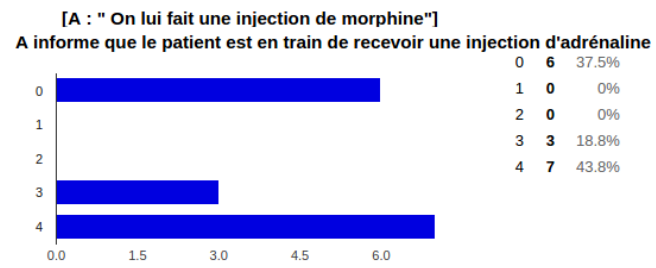

Figure 8. Using a word for another

Last, we observe that some meanings lead to separate groups in terms of interpretation: some meanings are totally rejected (rated 0) by most participants, while considered as totally acceptable (rated 4) by others (figure 9). We can consider that such person interacting together would have difficulties to understand each other.

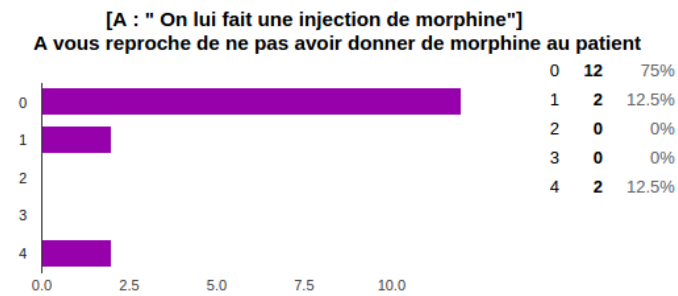

Figure 9. Meanings that seem rejected

\section{Conclusion}

Our communication model supports error generation in four stages of the communication process:

1) Upon message construction: alternative meanings are given to produce misunderstandings. The proposal is based on data of medical field training.

2) During message transport: the environment distorts the message depending on the communication channels that connects the sender to all possible listeners. The alteration differs from one agent to the other. This allows us to simulate non-understanding errors. 
3) Upon message reception: the agent can ignore a message, leading to errors of type "no answer".

4) When processing the message, if the recipient agent did not ignore the message, it chooses between acting or asking for a repetition to the sender. If acting with an incomplete message or with on alternative meaning, misinterpretation errors can appear.

This model has two originalities: it uses alternative meanings (perlocutionary acts) and it represents multi-modality in the interaction.

The main limit of the current model is that alternative meanings are not automatically produced. We used a questionnaire to validate, in a subjective manner and with a limited number of participants, the errors that our model produces. One medium-term goal is to use the probability of apparition of the misunderstanding as reported in our experiment to parameter the probability of error and to automatically generate alternative meanings.

Modelisation of error among a team is a difficult problem that cannont be narrowed down to communication errors. That is why, in our future work, we plan to combine this communication model with a model of collective activity to describe and reason about the other agents behaviours and a decision model for agents to generate erroneous behaviours in this collective activity. This will allow us to widden our model of error among the team with dimensions such as errors in communication decision: when, what and whom to communicate.

\section{Acknowledgements}

The VICTEAMS project (ANR-14-CE24-0027) has received funding from French National Research Agency (ANR), the French Defence Procurement Agency (DGA) and is labelled by the Labex MS2T. The authors would like to thank the Picardie region and the European Regional Development Fund (ERDF) 2014/2020 for the funding of this work.

\section{References}

[1] R. S. Lazarus and S. Folkman, "Stress," Appraisal, and coping, vol. 725, 1984

[2] J. E. Driskell and E. Salas, Stress and human performance. Psychology Press, 2013.

[3] E. Hollnagel, "Barriers and accident prevention," 2004.

[4] L. Cuvelier, "De la gestion des risques à la gestion des ressources de l'activité: étude de la résilience en anesthésie pédiatrique," Ph.D. dissertation, Conservatoire national des arts et metiers-CNAM, 2011.

[5] R. Flin, J. Winter, and M. R. Cakil Sarac, "Human factors in patient safety: review of topics and tools," World Health, p. 2, 2009.

[6] C. E. Shannon and W. Weaver, "The mathematical theory of communication," 1945.

[7] S. McRoy, "Preface: Detecting, repairing and preventing human-machine miscommunication," International Journal of HumanComputer Studies, vol. 48, no. 5, pp. 547-552, 1998.

[8] F. Schulz von Thun, "Miteinander reden: Störungen und klärungen," Rowohlt, Reinbek, 1981.
[9] M. St Pierre, G. Hofinger, and C. Buerschaper, Crisis Management in Acute Care Settings. Springer Berlin Heidelberg, 2008.

[10] J. L. Austin, "How to do things with words," 1962.

[11] J. R. Searle, Speech acts: An essay in the philosophy of language. Cambridge university press, 1969.

[12] F. Fipa, "specification part 2: Agent communication language," Technical report, FIPA-Foundation for Intelligent Physical Agents, Tech. Rep., 1997.

[13] C. Rich and C. L. Sidner, "Collagen: A collaboration manager for software interface agents," User Modeling and User-Adapted Interaction, vol. 8, no. 3-4, pp. 315-350, 1998.

[14] J. Allen, G. Ferguson, M. Swift, A. Stent, S. Stoness, L. Galescu, N. Chambers, E. Campana et al., "Two diverse systems built using generic components for spoken dialogue:(recent progress on trips)," in Proceedings of the ACL 2005 on Interactive poster and demonstration sessions. Association for Computational Linguistics, 2005, pp. 8588.

[15] M. Barange, "Task-oriented communicative capabilities of agents in collaborative virtual environments for training," Ph.D. dissertation, Université de Bretagne Occidentale (UBO), Brest, 2015.

[16] J. Shin, S. S. Narayanan, L. Gerber, A. Kazemzadeh, D. Byrd et al., "Analysis of user behavior under error conditions in spoken dialogs." in INTERSPEECH, 2002.

[17] C. Baber and K. S. Hone, "Modelling error recovery and repair in automatic speech recognition," International Journal of Man-Machine Studies, vol. 39, no. 3, pp. 495-515, 1993.

[18] D. Bohus, "Error awareness and recovery in task-oriented spoken dialogue systems," Retrieved at, p. 49, 2004.

[19] L. Ardissono, G. Boella, and R. Damiano, "A computational model of misunderstandings in agent communication," in $A I^{*} I A$ 97: Advances in Artificial Intelligence. Springer, 1997, pp. 48-59.

[20] E. Platon, N. Sabouret, and S. Honiden, "Overhearing and direct interactions: Point of view of an active environment," in Environments for multi-agent systems II. Springer, 2005, pp. 121-138.

[21] H. Bunt, "The dit++ taxonomy for functional dialogue markup," in AAMAS 2009 Workshop, Towards a Standard Markup Language for Embodied Dialogue Acts, 2009, pp. 13-24.

[22] E. Prud'Hommeaux, A. Seaborne et al., "Sparql query language for rdf," W3C recommendation, vol. 15, 2008.

[23] G. Klyne and J. J. Carroll, "Resource description framework (rdf): Concepts and abstract syntax," 2006.

[24] F. Balbo, N. Maudet, and J. Saunier, "Interactions opportunistes par l'écoute flottante," Actes des Journées Francophones sur les Systèmes Multi-Agents, vol. 2004, pp. 265-270, 2004.

[25] J. A. Easterbrook, "The effect of emotion on cue utilization and the organization of behavior." Psychological review, vol. 66, no. 3, p. $183,1959$.

[26] L. Mazuel and N. Sabouret, Semantic relatedness measure using object properties in an ontology. Springer, 2008. 